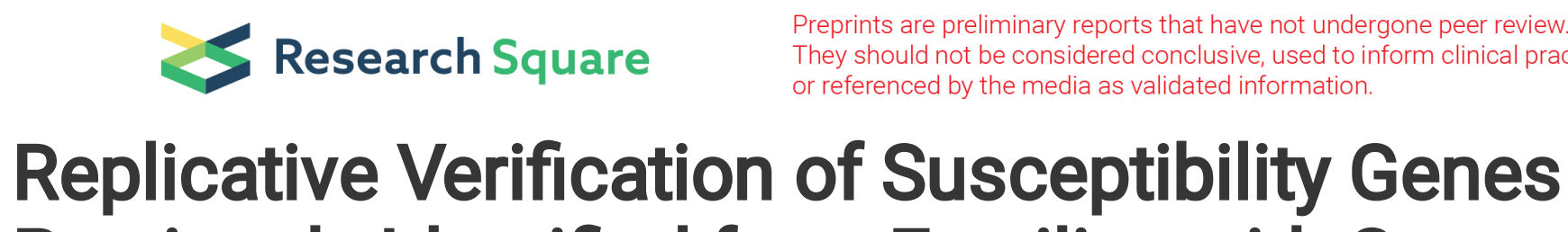 \\ Previously Identified from Families with Segregating Developmental Dysplasia of the Hip
}

\section{Xiaowen Xu}

Shengjing Hospital of China Medical University

\section{Binbin Wang}

National Research Institute for Family Planning

\section{Yufan Chen}

Shengjing Hospital of China Medical University

Weizheng Zhou

Shengjing Hospital of China Medical University

Lianyong Li ( $\sim$ loyo_Idy@163.com )

\section{Research}

Keywords: Developmental dysplasia of the hip, Sanger sequencing, Sporadic sample verification

Posted Date: July 27th, 2020

DOI: https://doi.org/10.21203/rs.3.rs-46911/v1

License: (c) (1) This work is licensed under a Creative Commons Attribution 4.0 International License.

Read Full License 


\section{Abstract}

Background: Developmental dysplasia of the hip (DDH) is a complex hip joint deformity with effects ranging from acetabulum malformation to irreversible hip dislocation. Previous studies suggest a significant association of four variations, Teneurin transmembrane protein 3 (TENM3) (chr4:183721398), Heparan sulfate proteoglycan 2 (HSPG2) (chr1:22201470), ATPase plasma membrane $\mathrm{Ca}^{2+}$ transporting 4 (ATP2B4) (chr1:203682345), and Prostaglandin F receptor (PTGFR) (chr1:79002214), with DDH susceptibility in families with segregating DDH. However, the association was not validated in sporadic cases and remains controversial. To confirm the association of the reported variations in these four genes with DDH, we conducted replicative verification in 250 sporadic samples with DDH from a Chinese Han population.

Methods: We conducted Sanger sequencing after amplifying the variation sites. The results were compared with the reference sequence from the GRCh37 assembly in UCSC (http://genome.ucsc.edu).

Results: Replication analysis of 250 sporadic samples by Sanger sequencing indicated that the four variations, TENM3 (chr4:183721398), HSPG2 (chr1:22201470), ATP2B4 (chr1:203682345), and PTGFR (chr1:79002214), were not associated with the susceptibility to DDH in the Chinese Han population.

Conclusions: Further studies should be performed to identify other variations of these four genes that are potentially associated with DDH by whole-exome sequencing and the results should be verified in different populations.

\section{Background}

Developmental dysplasia of the hip (DDH) is a common congenital malformation,which is related to hip osteoarthritis at an early age [1,2]. DDH occurs at a rate of approximately 5 per 1000 live births [3]; diagnosis and therapy at an early stage are crucial for avoiding serious complications.

The etiology of DDH is multifactorial and includes both genetic and environmental factors [4]. Studies have shown that breech presentation, oligohydramnios, female gender, higher birth weight, and primiparity are environmental factors affecting DDH [4]. Monozygotic twins were shown to have a higher risk of DDH than dizygotic twins [5]. Additionally, people who have first-degree relatives with DDH are susceptible to a 12-fold higher risk of developing the condition [6], indicating the important role of genetic factors. However, the pathogenic genes causing DDH are yet to be identified [6]. Several genes, including WNT inhibitory factor 1 (WIF1), Collagen type XI alpha 2 chain (COL11A2), and Pregnancy-associated plasma protein A2 (PAPPA2), were shown to increase susceptibility to DDH through candidate association studies in one population [7-10]. However, these results could not be confirmed in other populations.

DDH is a polygenic disease with complex genetic causes [10]. A series of studies on C-X3-C motif chemokine receptor 1 (CX3CR1) provided methods for identifying DDH-related genes. Feldman et al. [11] 
found that the variant rs3732378 in CX3CR1 causes a threonine to methionine amino acid change in the coding sequence. This variation was shared by four American DDH-affected family members, according to whole-exome sequencing linkage analysis. Li et al. [12] identified the variant rs3732378 in CX3CR1 in a case-control study of thousands of sporadic DDH samples. Subsequent studies showed that ablation of CX3CR1 affects acetabular morphology and gait in CX3CR1 knock-out mice [11]. PAPPA2 has also been shown to be related with $\operatorname{DDH}[9,13]$.

Recently, several possible variations in TENM3, ATP2B4, HSPG2, and PTGFR, which are related to DDH, were detected by genome-wide linkage analysis of three families $[5,14,15]$. TENM3 (chr4:183721398) was found to contain a novel $\mathrm{A}$ to $\mathrm{C}$ nucleotide alteration, causing a glutamine to proline change in a four-generation pedigree from Philadelphia, through linkage analysis [5]. Previous studies revealed two heterozygous rare coding variants in HSPG2 (chr1:22201470) and ATP2B4 (chr1:203682345) in a Saudi family with DDH and found that HSPG2 regulates ATP2B4 expression through transcription factors, as determined by in silico analysis [14]. A termination codon variation (c.922C $>\mathrm{T}$ ) in PTGFR

(chr1:79002214), which was located in exon $2 / 4$ of chromosome 1 , was detected in a Chinese family with $\mathrm{DDH}[15]$.

However, whether the variations TENM3 (chr4:183721398), HSPG2 (chr1:22201470), ATP2B4 (chr1:203682345), and PTGFR (chr1:79002214) are related to DDH in sporadic samples remains unclear. Therefore, this study was conducted to identify the association between these four variations and DDH in a large sample of sporadic cases with DDH in a Chinese Han population.

\section{Methods}

\subsection{Subjects}

A total of 250 patients with sporadic DDH from a Chinese Han population participated in this study. All subjects were diagnosed at the authors' institution. The patients were diagnosed with unilateral or bilateral DDH by two experienced pediatric orthopedics experts according to their medical history and based on the results of physical examination and pelvic radiograph. Patients with syndromic or neuromuscular hip dislocation were excluded. This study was approved by the Medical Ethic Committee of the authors' institution. Informed written consent was obtained from the parents or guardians of all patients.

\subsection{Sanger sequencing}

Blood samples were collected for genomic DNA extraction using the QIAamp DNA Blood kit (QIAGEN, Hilden, Germany) according to the manufacturer's protocol. The concentration and quality of DNA were assessed with a Nanodrop spectrophotometer (Thermo, Delaware, USA). Variations in TENM3, ATP2B4, HSPG2, and PTGFR were obtained from a previous family linkage analysis $[5,14,15]$ (Table 1). Primers for polymerase chain reaction (PCR) were designed using Primer 5 software and synthesized by Sangon Biotech (Shanghai, China) (Table 1). The reaction system was added according to the instructions of 
KOD-Multi \& Epi kit (TOYOBO, Osaka, Japan) for TENM3. The amplification conditions were as follows: $94{ }^{\circ} \mathrm{C}$ for 2 min for pre-denaturation, then $98^{\circ} \mathrm{C}$ for $10 \mathrm{~s}, 68^{\circ} \mathrm{C}$ for $15 \mathrm{~s}$, and $68^{\circ} \mathrm{C}$ for 2 min for 40 cycles. And the amplification conditions used for ATP2B4, HSPG2, and PTGFR were $95^{\circ} \mathrm{C}$ for 5 min for predenaturation, then $94^{\circ} \mathrm{C}$ for $30 \mathrm{~s}, 58^{\circ} \mathrm{C}$ for $30 \mathrm{~s}$, and $72{ }^{\circ} \mathrm{C}$ for $60 \mathrm{~s}$ for 38 cycles, and followed by $72{ }^{\circ} \mathrm{C}$ for $10 \mathrm{~min}$. The products of Sanger sequencing were analyzed using an ABI 3730XL Genetic Analyzer (Applied Biosystems, Foster City, USA). The results were compared with the reference sequence derived from GRCh37 assembly of UCSC (http://genome.ucsc.edu).

Table 1. Primer sequences

\begin{tabular}{cccc}
\hline Gene & Variation location & Forward Primer & Reverse Primer \\
\hline \multirow{2}{*}{ TENM3 } & chr4:183721398 & 5'-GCTCAACAACGCCTTCTACC-3' & 5'-TGTGGGTCGTAACAGCAGTC-3' \\
HSPG2 & chr1:22201470 & 5'-GGAAGGGTGATGGTTTGGAG-3' & 5'-CAGAAAGATGGCAGTGGGAG-3' \\
ATP2B4 & chr1:203682345 & 5'-AAAGCAGGGCCTGAATGGT-3' & 5'-GAGGTTGCAGTGAGCTGAGAT-3' \\
PTGFR & chr1:79002214 & 5'-GTCTCCATCAGTGTTTCTGCTAT & 5'-GAATTTTCCAGTTAGGCTACAG \\
& & C-3' & GTA-3' \\
\hline
\end{tabular}

\section{Results}

The four variations previously identified by linkage analysis of different families with DDH were not detected by Sanger sequencing of the 250 sporadic samples. These results indicate the variations in the pedigree segregating DDH in TENM3 (chr4:183721398), HSPG2 (chr1:22201470), ATP2B4 (chr1:203682345), and PTGFR (chr1:79002214) are not associated with susceptibility to DDH in the Chinese Han population.

\section{Discussion}

Previous studies showed that DDH is a polygenic malformation disease [4]. And most of the DDH gene studies are candidate gene association studies at present, but family linkage analysis can find main effect genes of DDH. Linkage analysis studies can detect variations that are potentially related to DDH through pedigree analysis, although the results require confirmation through a series of experiments using different populations and animal models. Feldman et al [11] found that CX3CR1 co-segregated with DDH families by linkage analysis and Li et al [12] verified the pathogenicity of the gene in sporadic cases subsequently, which identified a pathogenic gene systematically. The possible pathogenic genes currently 
identified by family linkage analysis are TENM3, HSPG2, ATP2B4 and PTGFR gene, but none of them have been validated in the disseminated DDH.

Teneurin 3 belongs to a highly conserved family of proteins and is necessary for various functions, including cell adhesion, cytoskeleton interaction, and calcium binding $[16,17]$. Previous studies revealed a novel mutation in TENM3, which co-segregated in all severely affected members in a three-generation family from Philadelphia. This mutation also delayed development of the left acetabulum and left glenoid fossa, as observed by Alcian blue staining in 8-week-old knock-in mutant mice [5]. Feldeman et al found that MMP13, which is related to chondrogenesis [18], was overexpressed in femur-derived bone cells of knock-in mice [5]. Previous studies showed that TENM3 is related to cartilage formation and expressed in prechondrogenic mesenchymal cells.

HSPG2 encodes the perlecan protein, a heparan sulfate proteoglycan [19], which localizes in basement membranes, vascular structures, cartilage, and osteogenic tissues [20] and participates in cellular proliferation, differentiation, and migration [21]. In vitro studies showed that perlecan regulates chondrocyte differentiation, which plays an important role in cartilage development [22]. Previous studies showed that Safranin-0 staining of the cartilage and expression of the typical collagen network were decreased in perlecan-deficient mice [20], which may have affected the occurrence of DDH. Therefore, HSPG2 may cause DDH by affecting cartilage growth.

Heterozygous variation in ATP2B4 was observed in a Saudi family pedigree, with HSPG2 shared by all three affected individuals in the family, as revealed by whole exome sequencing [14]. Using in silico analysis, Basit et al found that ATP2B4 expression was regulated by HSPG2 and involved various transcription factors [15]. Previous studies showed that ATP2B4 participated in the regulation of bone homeostasis through calcium signaling [23]. The expression level of ATP2B4 was increased during cellular senescence in human mesenchymal stem cells; these cells show the potential to differentiate into chondrocytes, which are components of the hip [24, 25]. Thus, ATP2B4 may play an important role in hip joint formation.

PTGFR, also known as FP, belongs to the G protein-coupled receptor family of seven transmembranespanning receptors. PTGFR plays an important role in chondrocyte differentiation and cartilage development [26-28]. PGF2a exerts its biological activities by binding to its receptor, PTGFR [29]. Previous studies showed that PGF2a stimulated the expression of cartilage marker genes in a rat cell line and human articular chondrocytes $[29,30]$. Studies also revealed that PTGFR participates in chondrocyte hypertrophy differentiation by regulating Bmp signaling [27]. Therefore, PTGFR may affect hip joint development as a receptor of PGF2a, which promotes cartilage formation.

In this study, we did not observe an association between these four variations and DDH; however, the association between other sites in these four genes and DDH remains unclear. The 250 sporadic cases evaluated in this study were from a Chinese Han population, indicating the possibility of a populationspecific result. The relationship between these variations and DDH in other populations requires further analysis. 


\section{Conclusions}

Our replication study indicated that no association exists between the four previously reported variations in TENM3 (chr4:183721398), HSPG2 (chr1:22201470), ATP2B4 (chr1:203682345), and PTGFR (chr1:79002214) and DDH in a Chinese Han population. However, other sites in these genes were not evaluated. Further studies are needed to identify other variations in these genes that are potentially associated with DDH by whole-exome sequencing, followed by verification of the associations in different populations.

\section{Abbreviations}

$\mathrm{DDH}$

Developmental dysplasia of the hip

TENM3

Teneurin transmembrane protein 3

HSPG2

Heparan sulfate proteoglycan 2

ATP2B4

ATPase plasma membrane $\mathrm{Ca}^{2+}$ transporting 4

PTGFR

Prostaglandin F receptor

WES

Whole-exome sequencing

WIF1

WNT inhibitory factor 1

COL11A2

Collagen type XI alpha 2 chain

CX3CR1

C-X3-C motif chemokine receptor 1

PAPPA2

Pregnancy-associated plasma protein A2

PCR

Polymerase chain reaction

GDF5

Growth differentiation factor 5

MMP13

Matrix metallopeptidase 13

\section{Declarations}




\section{Ethics approval and consent to participate}

This study was approved by the Medical Ethic Committee of Shengjing Hospital Affiliated to China Medical University (ID:2016PS092H). Informed written consent was obtained from all patients' parents or guardians.

\section{Consent for publication}

Consents for publication were obtained from participants parents.

\section{Availability of data and materials}

The datasets used and analyzed in this present study are available from the corresponding author on reasonable request.

\section{Competing interests}

The authors declare that they have no competing interests.

\section{Funding}

The study was supported by the National Natural Science Foundation of China (grant no. 81772296).

\section{Authors' contributions}

$\mathrm{LL}$ and BW contributed to the design of the study. YC and WZ helped conduct this study. XX contributed significantly to data analysis and manuscript writing. All authors have read and approve the final submitted manuscript.

\section{Acknowledgements}

We thank participant children and their guardians for their active participation in our study.

\section{References}

1. Sun Y, You Y, Jiang W, Zhai Z, Dai K. 3D-bioprinting a genetically inspired cartilage scaffold with GDF5-conjugated BMSC-laden hydrogel and polymer for cartilage repair. Theranostics. 2019;9(23):6949-6961. doi: 10.7150/thno.38061.

2. Basit S, Alharby E, Albalawi AM, Khoshhal KI. Whole genome SNP genotyping in a family segregating developmental dysplasia of the hip detected runs of homozygosity on chromosomes 15q13.3 and 19p13.2. Congenit Anom (Kyoto). 2018;58(2):56-61. doi: 10.1111/cga.12235. 
3. Bialik V, Bialik GM, Blazer S, Sujov P, Wiener F, Berant M. Developmental dysplasia of the hip: a new approach to incidence. Pediatrics. 1999;103(1):93-9.

4. Shi D, Dai J, Ikegawa S, Jiang Q. Genetic study on developmental dysplasia of the hip. Eur J Clin Invest. 2012;42(10):1121-5. doi:10.1111/j.1365-2362.2012.02682.x.

5. Feldman G, Kappes D, Mookerjee-Basu J, Freeman T, Fertala A, Parvizi J. Novel mutation in Teneurin 3 found to co-segregate in all affecteds in a multi-generation family with developmental dysplasia of the hip. J Orthop Res. 2019;37(1):171-180.

6. Stevenson DA, Mineau G, Kerber RA, Viskochil DH, Schaefer C, Roach JW. Familial predisposition to developmental dysplasia of the hip. J Pediatr Orthop. 2009;29(5):463-6.

7. Sun Y, You Y, Dai K, Zhang J, Yan M, Zhang Y. Genetic variant of WIF1 gene is functionally associated with developmental dysplasia of the hip in Han Chinese population. Sci Rep. 2019;9(1):285.

8. Xu R, Jiang X, Lu J, Wang K, Sun Y, Zhang Y. Genetic variant of COL11A2 gene is functionally associated with developmental dysplasia of the hip in Chinese Han population. Aging (Albany NY). $2020 ; 12$.

9. Jia J, Li L, Zhao Q, Zhang L, Ru J, Liu X, et al. Association of a single nucleotide polymorphism in pregnancy-associated plasma protein-A2 with developmental dysplasia of the hip: a case-control study. Osteoarthritis Cartilage. 2012;20(1):60-3.

10. Harsanyi S, Zamborsky R, Krajciova L, Kokavec M, Danisovic L. Developmental Dysplasia of the Hip: A Review of Etiopathogenesis, Risk Factors, and Genetic Aspects. Medicina (Kaunas). 2020;56(4):E153.

11. Feldman G, Offemaria A, Sawan H, Parvizi J, Freeman TA. A murine model for developmental dysplasia of the hip: ablation of CX3CR1 affects acetabular morphology and gait. J Transl Med. 2017;15(1):233.

12. Li L, Wang X, Zhao Q, Wang E, Wang L, Cheng J, et al. CX3CR1 polymorphisms associated with an increased risk of developmental dysplasia of the hip in human. J Orthop Res. 2017;35(2):377-380.

13. Chen Y, Li L, Wang E, Zhang L, Zhao Q. Abnormal expression of Pappa2 gene may indirectly affect mouse hip development through the IGF signaling pathway. Endocrine. 2019;65(2):440-450.

14. Basit S, Albalawi AM, Alharby E, Khoshhal KI. Exome sequencing identified rare variants in genes HSPG2 and ATP2B4 in a family segregating developmental dysplasia of the hip. BMC Med Genet. 2017;18(1):34.

15. Wang Sun, Jiao Qin, Zhou Zaiwei, Wu Jing, Fan Linyan, Chen Mengjie, et al. Whole-exome analysis in pedigree of developmental dysplasia of the hip (in Chinese). Chin J Pediatr Surg. 2018;39(3).

16. Bastías-Candia S, Martínez M, Zolezzi JM, Inestrosa NC. Wnt Signaling Upregulates Teneurin-3 Expression via Canonical and Non-canonical Wnt Pathway Crosstalk. Front Neurosci. 2019;13:505.

17. Tucker RP, Kenzelmann D, Trzebiatowska A, Chiquet-Ehrismann R. Teneurins: transmembrane proteins with fundamental roles in development. Int J Biochem Cell Biol. 2007;39(2):292-7. 
18. Bertrand J, Kräft T, Gronau T, Sherwood J, Rutsch F, Lioté F, et al. BCP crystals promote chondrocyte hypertrophic differentiation in OA cartilage by sequestering Wnt3a. Ann Rheum Dis. 2020;79(7):975984.

19. Pei S, Parthasarathy S, Parajuli A, Martinez J, Lv M, Jiang S, et al. Perlecan/Hspg2 deficiency impairs bone's calcium signaling and associated transcriptome in response to mechanical loading. Bone. 2020;131:115078.

20. Costell M, Gustafsson E, Aszódi A, Mörgelin M, Bloch W, Hunziker E, et al. Perlecan maintains the integrity of cartilage and some basement membranes. J Cell Biol. 1999;147(5):1109-22.

21. Hayes AJ, Gibson MA, Shu C, Melrose J. Confocal microscopy demonstrates association of LTBP-2 in fibrillin-1 microfibrils and colocalisation with perlecan in the disc cell pericellular matrix. Tissue Cell. 2014;46(3):185-97.

22. French MM, Smith SE, Akanbi K, Sanford T, Hecht J, Farach-Carson MC, et al. Expression of the heparan sulfate proteoglycan, perlecan, during mouse embryogenesis and perlecan chondrogenic activity in vitro. J Cell Biol. 1999;145(5):1103-15.

23. Kim HJ, Prasad V, Hyung SW, Lee ZH, Lee SW, Bhargava A, et al. Plasma membrane calcium ATPase regulates bone mass by fine-tuning osteoclast differentiation and survival. J Cell Biol. 2012;199(7):1145-58.

24. Yoo JK, Choi SJ, Kim JK. Expression profiles of subtracted mRNAs during cellular senescence in human mesenchymal stem cells derived from bone marrow. Exp Gerontol. 2013;48(5):464-71.

25. Mang T, Kleinschmidt-Dörr K, Ploeger F, Lindemann S, Gigout A. The GDF-5 mutant M1673 exerts robust anabolic and anti-catabolic effects in chondrocytes. J Cell Mol Med. 2020;24(13):7141-50.

26. Lee K, Lee SH, Kim TH. The Biology of Prostaglandins and Their Role as a Target for Allergic Airway Disease Therapy. Int J Mol Sci. 2020;21(5):1851.

27. Kim J, Shim M. Prostaglandin F2a receptor (FP) signaling regulates Bmp signaling and promotes chondrocyte differentiation. Biochim Biophys Acta. 2015;1853(2):500-12.

28. Milne SA, Jabbour HN. Prostaglandin (PG) F(2alpha) receptor expression and signaling in human endometrium: role of PGF(2alpha) in epithelial cell proliferation. J Clin Endocrinol Metab. 2003;88(4):1825-32.

29. Lowe GN, Fu YH, McDougall S, Polendo R, Williams A, Benya PD, et al. Effects of prostaglandins on deoxyribonucleic acid and aggrecan synthesis in the RCJ 3.1C5.18 chondrocyte cell line: role of second messengers. Endocrinology. 1996;137(6):2208-16.

30. Jakob M, Démarteau O, Suetterlin R, Heberer M, Martin I. Chondrogenesis of expanded adult human articular chondrocytes is enhanced by specific prostaglandins. Rheumatology (Oxford). $2004 ; 43(7): 852-7$. 